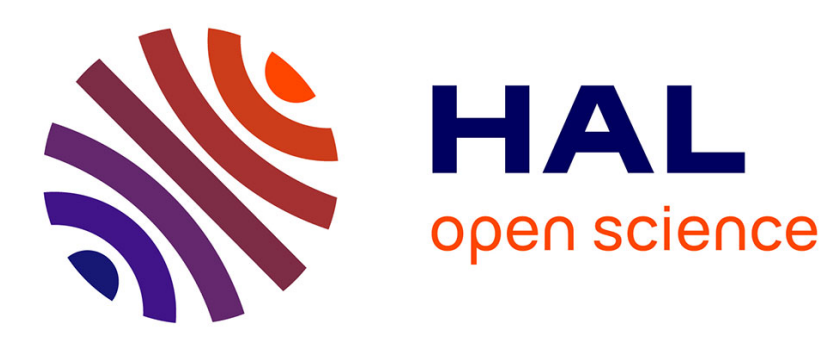

\title{
Clustering in Stochastic Asynchronous Algorithms for Distributed Simulations
}

\author{
Anatoly Manita, François Simonot
}

\section{To cite this version:}

Anatoly Manita, François Simonot. Clustering in Stochastic Asynchronous Algorithms for Distributed Simulations. Lecture Notes in Computer Science, 2005, Stochastic Algorithms: Foundations and Applications, Lecture Notes in Computer Science (3777), pp.26-37. 10.1007/11571155_3 . inria00000783

\section{HAL Id: inria-00000783 https://hal.inria.fr/inria-00000783}

Submitted on 18 Nov 2005

HAL is a multi-disciplinary open access archive for the deposit and dissemination of scientific research documents, whether they are published or not. The documents may come from teaching and research institutions in France or abroad, or from public or private research centers.
L'archive ouverte pluridisciplinaire HAL, est destinée au dépôt et à la diffusion de documents scientifiques de niveau recherche, publiés ou non, émanant des établissements d'enseignement et de recherche français ou étrangers, des laboratoires publics ou privés. 


\title{
On the cascade rollback synchronization
}

\author{
Anatoli Manita* François Simonot ${ }^{\dagger}$
}

15th March 2005

\begin{abstract}
We consider a cascade model of $N$ different processors performing a distributed parallel simulation. The main goal of the study is to show that the long-time dynamics of the system has a cluster behavior. To attack this problem we combine two methods: stochastic comparison and Foster-Lyapunov functions.
\end{abstract}

\section{Introduction}

The present paper contains a probabilistic analysis of some mathematical model of asynchronous algorithm for parallel simulation. For the detailed discussion of synchronization issues in parallel and distributed algorithms we refer to $[2,14]$. Here we give only a brief description of the problem. In large-scale parallel computation it is necessary to coordinate the activities of different processor which are working together on some common task. Usually such coordination is implemented by using a so-called message-passing system. This means that a processor shares data with other processors by sending timestamped messages. Between sending or receiving the messages the processors work independently. It can be happened that till the moment of receiving of a message some processor can proceed farther in performing its program than the value of timestamp indicated in this newly received message; in this case the state of the processor should be rolled back to the indicated value. It is clear that due to possible rollbacks the mean speed of a given processor

${ }^{*}$ The work of this author is supported by Russian Foundation of Basic Research (grant 02-01-00945). Postal address: Faculty of Mathematics and Mechanics, Moscow State University, 119992, Moscow, Russia. E-mail: manita@mech.math.msu.su

${ }^{\dagger}$ IECN, Université Henri Poincaré Nancy I, Esstin, 2, Rue J. Lamour, 54500 Vandoeuvre, France. E-mail: francois.simonot@esstin.uhp-nancy.fr 
in the computing network will be lower than its proper speed. One of the most important performance characteristics of the system is the progress of the computing network on large time intervals.

Probabilistic models for such system are studied already for twenty years. From the probabilistic point of view these models consists of many relatively independent components which synchronize from time to time their states according to some special algorithm. The detailed review of all existing publications is out of range of this paper. We would like to mention only that the bibliography on this subject consists mostly of two group of papers. The first group of publication $[3,10,11,9,6]$ are devoted to the case of two processors. The paper [3] is of special interest since it contains an exhaustive basic analysis of the two-dimensional model and had a big influence on the further research. The case of many processors was studied in $[5,6,7,15,12,13]$. An important difference of such models from two-dimensional case is that in a realistic model with more than two processors one message can provoke a multiple rollback of a chain of processors. Since the multi-dimensional model is much more complicated for a rigorous study, in the above papers the authors deal with the set of identical processors and their mathematical results are contained in preparatory sections before large numerical simulations.

It should be noted also that probabilistic models with synchronization mechanism are interesting also for modelling database systems (see for example, [14]). Moreover, now synchronization-like interactions are considered as well in the framework of interaction particle systems $[16,17,18]$.

The model considered in the present paper is of special interest for the following reasons. We deals with a nonhomogeneus model consisting of several different processors. We consider case of message-passing topology other from the topology of complete graph which was considered in all previous papers. Our main interest is the cascade model which pressupose a subordination between processors. We put forward a conjecture on the claster behavior of the system: processors can be divided into separated groups which are asymptotically independent and have their own proper performance characteristics. Our main goal is to justify this conjecture. One should point out that in the case of complete graph topology the claster decomposition into group is degenerated and, thus, not interesting.

We describe our model in terms of multi-dimensional continuous time Markov process. To get asymptotical performance characteristics of the model we combine two probabilistic methods (stochastic comparison and Foster-Lyapunov functions).

The paper is organized as follows. In Section 2 we give introduce a general continuous 
time Markov model and define a cascade model as a special subclass of the general model. In Section 3 we pass to the embedded Markov chain. Main problem is now to study a long-time behavior of Markov chain with highly nonhomogeneous transition probabilities. To do this we consider relative coordinates and find groups of processors whose evolution is ergodic (convergences to a steady state) in these relative coordinates. To our opinion the method of Foster-Lyapunov functions seems to be the only one to prove the stability in relative coordinates for the Markov chain under consideration. First of all in Section 5we start from the case of two processors $(N=2)$ and the analysis here is rather simple and similar to [3]. In the study of the three-dimensional case (Section 7) the main point is the proof of ergodicity. We propose an explicit construction of some nonsmooth FosterLyapunov function. Our construction is rather nontrivial as it can be seen by comparing with already existing explicit examples of Lyapunov functions (see [1]). All this analysis bring us to some conclusions presented in Section 8. This section contains decomposition into groups (clasters) in the case of cascade model with any number of processors $N$ and our main Conjection 14. We show that future progress in proving this conjecture may be related with progress in explicit construction of multi-dimensional Foster-Lyapunov functions. Analysis of random walks in $\mathbf{Z}_{+}^{n}$ (which was done in [1]) shows that, in general, this technical problem may be very difficult. In the next papers we hope to overcome these dificulties by using specific features of our concrete Markov processes.

Acknowledgements. The first author is very grateful to the team TRIO (INRIALorraine) and to l'Ecole des Mines de Nancy for their hospitality during his stay at Nancy in summer 2004 when the main results of this paper were obtained.

\section{Description of continuous time model}

\subsection{General model}

We present here some mathematical model for parallel computations. There are $N$ computing units (processors) working together on some common task. The state of a processor $k$ is described by an integer variable $x_{k} \in \mathbf{Z}$ which is called a local (or inner) time of the processor $k$ and has a meaning of amount of job done by the processor $k$ up to the given time moment.

Assume that the state $\left(x_{1}, x_{2}, \ldots, x_{N}\right)$ of the system evolves in continuous time $t \in \mathbf{R}_{+}$.

Any change of a state is posible only at some special random time instants. Namely, 
with any processor $k$ we associate a Poissonian flow $\Pi^{k}=\left\{0=\sigma_{0}^{k}<\sigma_{1}^{k}<\cdots<\sigma_{n}^{k}<\cdots\right\}$ with intensity $\lambda_{k}$ and with a pair $(k, l)$ of processors we associate a Poissonian flow $\Pi^{k l}=\left\{0=\sigma_{0}^{k l}<\sigma_{1}^{k l}<\cdots<\sigma_{n}^{k l}<\cdots\right\}$ with intensity $\beta_{k l}$. This means, for example, that $\left\{\sigma_{n}^{k}-\sigma_{n-1}^{k}\right\}_{n=1}^{\infty}$ is a sequence of independent exponentially distributed random variables with mean $\lambda_{k}^{-1}: \forall n=1,2, \ldots \quad \mathrm{P}\left\{\sigma_{n}^{k}-\sigma_{n-1}^{k}>0\right\}=\exp \left(-\lambda_{k} s\right)$, and similarly for the flows $\Pi^{k l}$. We assume also that all these flows $\Pi^{k}$ and $\Pi^{k l}$ are mutually independent.

Let us now define a stochastic process $\left(X(t)=\left(x_{1}(t), \ldots, x_{N}(t)\right), t \in \mathbf{R}_{+}\right)$on the state space $\mathbf{Z}^{N}$ according to the following rules.

1) At time instants $\sigma_{n}^{k}$ the processor $k$ increases its local time $x_{k}$ by 1: $x_{k}\left(\sigma_{n}^{k}+0\right)=$ $x_{k}\left(\sigma_{n}^{k}\right)+1$.

2) There is an exchange of information between different processors. At time instant $\sigma_{i}^{k l}$ the processor $k$ sends a message $m_{k l}^{\left(x_{k}\right)}$ to the processor $l$. We assume that messages reach their destination immediately. A message $m_{k l}^{\left(x_{k}\right)}$ coming to node $l$ from node $k$ contains an information about local time $x_{k}\left(\sigma_{i}^{k l}\right)=x_{k}$ of the sender $k$. If at the time instant $\sigma_{i}^{k l}$ (when the message $m_{k l}^{\left(x_{k}\right)}$ arrives to the node $\left.l\right)$ we have $x_{l}\left(\sigma_{i}^{k l}\right)>x_{k}\left(\sigma_{i}^{k l}\right)$ then the local time $x_{l}$ rolls back to the value $x_{k}: x_{l}\left(\sigma_{i}^{k l}+0\right)=x_{k}\left(\sigma_{i}^{k l}\right)$. Moreover, if the processor $l$ rolls back, then all messages sended by the processor $l$ during the time interval $\mathcal{I}=\left(\theta_{l}\left(x_{k}, \sigma_{i}^{k l}\right), \sigma_{i}^{k l}\right)$, where $\left.\theta_{l}(x, u):=\max \left\{s \leq u: x_{l}(s)=x, x_{l}(s+0)=x+1\right\}, \sigma_{i}^{k l}\right)$, should be eliminated. This can provoke a cascading rollback of local times for some subset of processors. For example, assume that there is a processor $q$ which received a message $m_{l q}^{\left(x_{l}^{\prime}\right)}$ at some time instant $s^{\prime} \in \mathcal{I}$ and $x_{q}\left(\sigma_{i}^{k l}\right)>x_{l}\left(s^{\prime}\right)=x_{l}^{\prime}$. Then the local clock of $q$ should be rolled back to the value $x_{l}\left(s^{\prime}\right): x_{q}\left(\sigma_{i}^{k l}+0\right)=x_{l}\left(s^{\prime}\right)$ and, moreover, all messages sended by $q$ during the interval $\mathcal{I}=\left(\theta_{q}\left(x_{l}\left(s^{\prime}\right), \sigma_{i}^{k l}\right), \sigma_{i}^{k l}\right)$ should be eliminated. And so on. Hence, at time instant $\sigma_{i}^{k l}$ a message from $k$ to $l$ can provoke a multiple rollback of processor $l, q, \ldots$ in the system.

\subsection{Cascade model}

From now we shall consider the following special subclass of the above general models.

A chain of processors $1,2, \ldots, N$ is called a cascade if any processor $j$ can send a message only to its right neigbour $j+1$. So noone sends messages to the processor 1 and the processor $N$ does not send any messages. In other words, $\beta_{i j} \neq 0 \Leftrightarrow(j=i+1)$. A message sended from $j$ to $j+1$ can provoke a cascading rool-back of processors $j+2, \ldots$ Recall that all above time intervals are exponentially distributed and supposed to be independent. Obviously, the stochastic process $X_{c}^{(N)}(t)=\left(x_{1}(t), \ldots, x_{N}(t)\right)$ is Markovian. A very important property is that any "truncated" marginal process $X_{c}^{\left(N_{1}\right)}(t)=\left(x_{1}(t), \ldots, x_{N_{1}}(t)\right)$, 
$N_{1} \leq N$, is also Markovian.

Assume that for any $j$ the following limit

$$
v_{j}^{*}=\lim _{t \rightarrow+\infty} \frac{x_{j}(t)}{t} \quad \text { (in probability) }
$$

exists. Then the numbers $v_{j}^{*}, j=1, \ldots, N$, characterize performance of the model. The main goal of the present paper is to prove existence of these limits and to calculate them.

Note that if we uniformly transform the absolute time scale $t=c s$, where $c>0$ is a constant and $s$ is a new absolute time scale, the performance characteristics (1) will not change.

\section{Definition of discrete time model}

Consider a sequence

$$
0=\tau_{0}<\tau_{1}<\tau_{2}<\cdots<\cdots
$$

of time moments when changes of local time at nodes may happen (we mean local time updates and moments of sending of messages). It is clear that $\left\{\tau_{r+1}-\tau_{r}\right\}_{r=0}^{\infty}$ is a sequence of independent identically distributed r.v. having exponential distribution with parameter

$$
Z=\sum_{i=1}^{N} \lambda_{i}+\sum_{i=1}^{N-1} \beta_{i, i+1}
$$

Observing the continuous time Markov process $\left(x_{1}(t), \ldots, x_{N}(t)\right)$ at time moments $\tau_{n}$ we get a so-called embedded discrete time Markov chain $\{X(n), n=0,1, \ldots\}$ with state space $\mathbf{Z}_{+}^{N}$. In the sequel we will be interested in the long-time behavior of the chain $\{X(n), n=0,1, \ldots\}$.

Transition probabilities. In the $\mathrm{MC}\{X(n), n=0,1, \ldots\}$ there are transitions produced by a free dynamics and transitions generated by rollbacks. By the free dynamics we mean updating of local times

$$
P\left\{X(n+1)=x+\mathbf{e}_{j} \mid X(n)=x\right\}=\lambda_{j} Z^{-1}, \quad j=1, \ldots, N .
$$


It is easy to see that if a state $x=\left(x_{1}, \ldots, x_{N}\right)$ is such that for some $j \quad x_{j}<x_{j+1}$ then a message sended from $j$ to $j+1$ produces a transition of the following form

$$
\left(x_{1}, \ldots, x_{j}, x_{j+1}, \ldots, x_{l}, x_{l+1}, \ldots, x_{N}\right) \rightarrow\left(x_{1}, \ldots, x_{j}, w_{j+1}, \ldots, w_{l}, x_{l+1}, \ldots, x_{N}\right)
$$

with probability

$$
Z^{-1} \beta_{j, j+1} \prod_{q=j+1}^{l-1} p\left(w_{q}, x_{q} ; w_{q+1}, x_{q+1}\right) \times\left(1-b_{l}\right)^{\min \left(x_{l}, x_{l+1}-1\right)-w_{l}+1}
$$

where

- sequence $\left(w_{j+1}, \ldots, w_{l}\right)$ is admissible in the following sense:

$$
j<l \leq N, \quad w_{j+1}=x_{j} \quad w_{q} \leq w_{q+1} \leq \min \left(x_{q}, x_{q+1}-1\right), \quad(j<q<l)
$$

- $p\left(w_{q}, x_{q} ; w_{q+1}, x_{q+1}\right)=b_{q}\left(1-b_{q}\right)^{w_{q+1}-w_{q}}$

- $b_{q}=\frac{\lambda_{q}}{\lambda_{q}+\beta_{q, q+1}}, q<N$.

Here $b_{q}$ is the probability of an event that processor $q$ in state $x_{q}$ sends at least one message to $q+1$ before updating its state $x_{q} \rightarrow x_{q}+1$. For $q=N$ we put $b_{N}=0$. So in the case $l=N$ the probability (3) takes the form

$$
Z^{-1} \beta_{j, j+1} \prod_{q=j+1}^{N-1} p\left(w_{q}, x_{q} ; w_{q+1}, x_{q+1}\right)
$$

Relative coordinates. Note that the first processor $x_{1}(t)$ evolves independently of other processors. It is useful to introduce new process $Y_{c}(t)=\left(y_{2}(t), \ldots, y_{N}(t)\right) \in \mathbf{Z}^{N-1}$ in relative coordinates as viewing by an observer sitting at the point $x_{1}(t)$ :

$$
y_{j}(t):=x_{j}(t)-x_{1}(t), \quad j=2, \ldots, N
$$

In a similar way we define $Y(n)=Y_{c}\left(\tau_{n}\right), n=0,1, \ldots$ The free dynamics produces the following transitions of $Y(n)$ :

$$
\begin{aligned}
P\left\{Y(n+1)=y+\mathbf{e}_{j} \mid Y(n)=y\right\} & =\lambda_{j} Z^{-1}, \quad j=2, \ldots, N, \\
P\left\{Y(n+1)=y-\sum_{j=2}^{N} \mathbf{e}_{j} \mid Y(n)=y\right\} & =\lambda_{1} Z^{-1} .
\end{aligned}
$$


Since rollback does not affect on the first processor the corresponding transitions have the same form and the same probabilities as (2) and (3).

\section{Stochastic monotonicity}

All statements of this section are valid for the both Markov processes $X_{c}^{(N)}(t), t \in \mathbf{R}_{+}$, and $X(n), n \in \mathbf{Z}_{+}$. For the sake of breavity we give here results only for the contionuous time model $X_{c}^{(N)}(t)$.

Theorem 1. Let us consider two cascade models (say $X_{c, 1}^{(n)}(t)$ and $X_{c, 2}^{(n)}(t)$ ) with processors $1,2, \ldots, n$ and parameters $\lambda_{1}, \ldots, \lambda_{n}$ and $\beta_{12}^{(1)}, \beta_{23}^{(1)}, \ldots, \beta_{n-1, n}^{(1)}$ for the first model $X_{c, 1}^{(n)}(t)$ and parameters $\lambda_{1}, \ldots, \lambda_{n}$ and $\beta_{12}^{(2)}, \beta_{23}^{(2)}, \ldots, \beta_{n-1, n}^{(2)}$ for the second model $X_{c, 2}^{(n)}(t)$. Assume that

$$
\beta_{i, i+1}^{(1)} \leq \beta_{i, i+1}^{(2)} \quad \forall i
$$

Then the first model $X_{c, 1}^{(n)}(t)$ stochastically dominates over the second model $X_{c, 2}^{(n)}(t)$ in the following sense: if $X_{c, 1}^{(n)}(0)=X_{c, 2}^{(n)}(0)$ then for any time moment $t$ we have ${ }^{1}$

$$
X_{c, 1}^{(n)}(t) \geq_{s t} X_{c, 2}^{(n)}(t)
$$

Proof may be given by an explicit coupling construction for the processes $X_{c, 1}^{(n)}(t)$ and $X_{c, 2}^{(n)}(t)$ on the same probability space. The following fact should be used: a Poisson flow with intensity $\beta_{12}^{(1)}$ can be obtained from a Poisson flow with intensity $\beta_{12}^{(2)}$ in which any point (independently from other) is killed with probability $1-\beta_{12}^{(1)} / \beta_{12}^{(2)}$.

Corollary 2 (Solid barriers). Fix some $1 \leq r_{1}<r_{2}<\cdots<r_{b}<n$ and consider two cascade models: $X_{c, 1}^{(n)}(t)$ with parameters $\left(\lambda_{1}, \ldots, \lambda_{n} ; \beta_{12}^{(1)}, \beta_{23}^{(1)}, \ldots, \beta_{n-1, n}^{(1)}\right)$ and $X_{c, 2}^{(n)}(t)$ with parameters $\left(\lambda_{1}, \ldots, \lambda_{n} ; \beta_{12}^{(2)}, \beta_{23}^{(2)}, \ldots, \beta_{n-1, n}^{(2)}\right)$, where

$$
\beta_{i, i+1}^{(2)}=\beta_{i, i+1}^{(1)} \quad \forall i \notin\left\{r_{1}, \ldots, r_{b}\right\}, \quad \beta_{i, i+1}^{(2)}=0 \quad \forall i \in\left\{r_{1}, \ldots, r_{b}\right\} .
$$

We can say that the model $X_{c, 2}^{(n)}(t)$ differs from the model $X_{c, 1}^{(n)}(t)$ by the presence of $b$ solid barriers between processors $r_{1}$ and $r_{1}+1, \ldots, r_{b}$ and $r_{b}+1$. Then by Theorem 1 we have

\footnotetext{
${ }^{1}$ It means that there exists a coupling $\left(\widetilde{X}_{1}^{(n)}(t, \omega), \widetilde{X}_{2}^{(n)}(t, \omega)\right)$ of stochastic processes $X_{1}^{(n)}(t)$ and $X_{2}^{(n)}(t)$ such that $P\left\{\omega: \widetilde{X}_{1}^{(n)}(t, \omega) \geq \widetilde{X}_{2}^{(n)}(t, \omega) \forall t\right\}=1$. If $w, z \in \mathbf{R}^{n}$ we say $w \geq z$ if $w_{i} \geq z_{i}$ for all $i=$ $1, \ldots, n$ (partial order).
} 
that

$$
X_{c, 1}^{(n)}(t) \leq_{\mathrm{s} t} X_{c, 2}^{(n)}(t)
$$

\section{Case $N=2$}

We start with the Markov chain $X_{c}^{(2)}(t)$. Since processor 1 works independently, the main interest has Markov chain $Y_{c}^{(2)}(t)=x_{2}(t)-x_{1}(t)$.

Taking in mind the remark at the end of Subsection 2.2, for breavity of notation let us rescale absolute time in such a way that $Z=1$. Then Markov chain $Y(n)$ has the following transition probabilities

$$
p_{i, i+1}=\lambda_{2}, \quad p_{i, i-1}=\lambda_{1}, \quad p_{i, 0}=\beta_{12}(i \geq 0), \quad p_{i, i}=\beta_{12}(i<0)
$$

and $p_{i, j}=0$ for any another pair $i, j$.

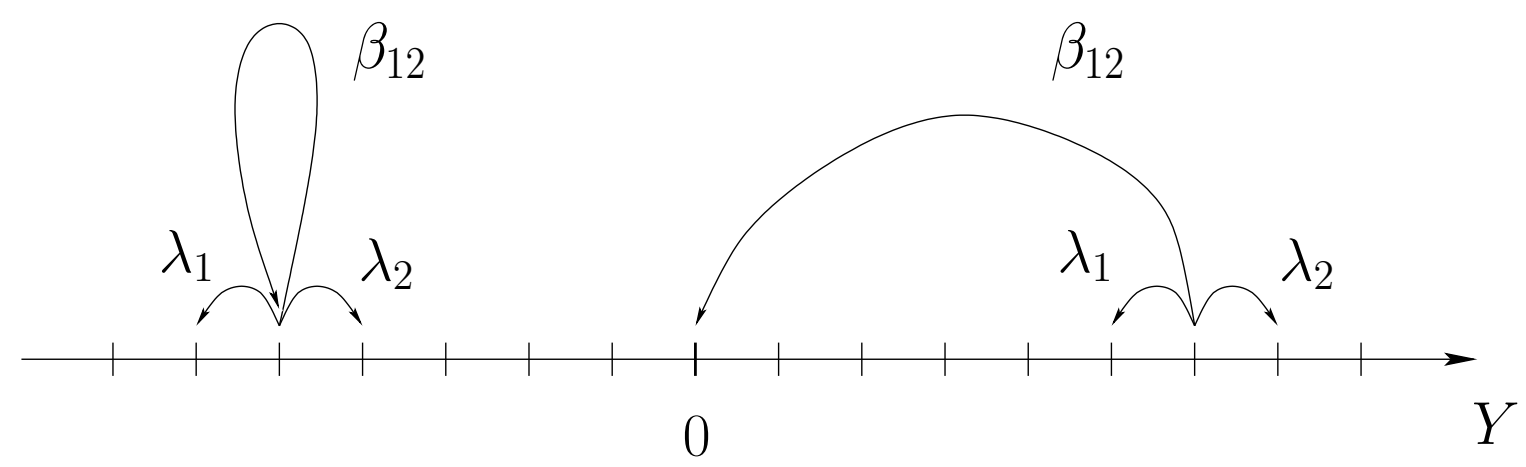

a

Theorem 3. If $\lambda_{1}<\lambda_{2}$ then Markov chain $Y(n)$ is ergodic. If $\lambda_{1}>\lambda_{2}$ then Markov chain $Y(n)$ is transient.

Corollary 4. If $\lambda_{1}<\lambda_{2}$ then $v_{1}^{*}=v_{2}^{*}=\lambda_{1}$. If $\lambda_{1}>\lambda_{2}$ then $v_{1}^{*}=\lambda_{1}$ and $v_{2}^{*}=\lambda_{2}$.

Proof. Markov chain $Y(n)$ is one-dimensional and its analysis is quite easy. To establish ergodicity under assumption $\lambda_{1}<\lambda_{2}$ we use the Foster-Lyapunov criterion (Theorem 17) with test function $f(y)=|y|, y \in \mathbf{Z}$. This implies that $x_{2}(t)-x_{1}(t)$ has a limit in distribution as $t \rightarrow \infty$. Recall that $x_{1}(t)$ is a Poissonian process hence the limit $v_{1}^{*}=$ $t^{-1} \lim _{t} x_{1}(t)=\lambda_{1}$ exists (in probability). It follows from this that $v_{2}^{*}=t^{-1} \lim _{t} x_{2}(t)=\lambda_{1}$.

Under assumption $\lambda_{1}>\lambda_{2}$ we get transiency by choosing the function $f(y)=\min \left(e^{\delta y}, 1\right)$, $y \in \mathbf{Z}$, where we fix sufficiently small $\delta>0$, and applying Theorem 18. So any trajectory 
of $Y(n)$ spends a finite time in any prefixed domain $\{y \geq C\}$ hence $x_{2}(t)-x_{1}(t) \rightarrow-\infty$ as $t \rightarrow \infty$ (a.s.). It means that after some time messages $1 \rightarrow 2$ can not produce a rollback anymore, so $x_{1}(t)$ and $x_{2}(t)$ become asymptotically independent and hence $v_{2}^{*}=t^{-1} \lim _{t} x_{2}(t)=\lambda_{2}$.

\section{Case $N=3$}

Theorem 5. Four situations are possible.

1. If $\lambda_{1}<\min \left(\lambda_{2}, \lambda_{3}\right)$ then $v_{1}^{*}=v_{2}^{*}=v_{3}^{*}=\lambda_{1}$.

2. If $\lambda_{2}>\lambda_{1}>\lambda_{3}$ then $v_{1}^{*}=v_{2}^{*}=\lambda_{1}, v_{3}^{*}=\lambda_{3}$.

3. If $\lambda_{2}<\min \left(\lambda_{1}, \lambda_{3}\right)$ then $v_{1}^{*}=\lambda_{1}, v_{2}^{*}=v_{3}^{*}=\lambda_{2}$.

4. If $\lambda_{1}>\lambda_{2}>\lambda_{3}$ then $v_{1}^{*}=\lambda_{1}, v_{2}^{*}=\lambda_{2}, v_{3}^{*}=\lambda_{3}$.

Items 2, 3 and 4 can be reduced in some sense to the results on the case $N=2$ (see Corollary 4). We prove them in the current section. Proof of item 1 is different and will be given in Sect. 7 .

Proof of Theorem 5 (items 2-4). We start from the item 2: $\lambda_{2}>\lambda_{1}>\lambda_{3}$. Since the first two processors are governed by the Markov chain $X_{c}^{(2)}(t)$ and do not depend on the state of processor 3 we apply Corollary 4 and conclude that $X_{c}^{(2)}(t)$ is ergodic and $v_{1}^{*}=v_{2}^{*}=\lambda_{1}$.

Let us compare the following two cascade models

$$
\begin{array}{ll}
X_{c}^{(3)}(t): & 1 \stackrel{\beta_{1,2}}{\longrightarrow} 2 \stackrel{\beta_{2,3}}{\longrightarrow} 3 \\
X_{c, 2}^{(3)}(t): & 1 \stackrel{\beta_{1,2}}{\longrightarrow} 2 \stackrel{0}{\longrightarrow} 3
\end{array}
$$

(parameters $\lambda_{1}, \lambda_{2}$ and $\lambda_{3}$ are the same for the both models $X_{c}^{(3)}(t)$ and $X_{c, 2}^{(3)}(t)$ ).

In the model $X_{c, 2}^{(3)}$ the groups of processors $\{1,2\}$ and $\{3\}$ evolve independently. Evidently, an asymptotic speed of processor 3 in the model $X_{c, 2}^{(3)}$ exists and is equal to $\lambda_{3}$. By Corollary $2 X_{c}^{(3)}(t) \leq_{s t} X_{c, 2}^{(3)}(t)$. Hence in the model $X_{c}^{(3)}$ an asymptotic speed of the 
processor 3 is not greater than $\lambda_{3}$. Since $\lambda_{3}<\lambda_{1}$ we conclude that there exists some time moment $T_{0}$ such that for $t \geq T_{0}$ in the model $X_{c}^{(3)}$ messages from 2 to 3 that roll back the processor 3 will be very "rare". So these rare rollbacks will be not essential for an asymptotical speed of the processor 3 . In other words, as $t \rightarrow \infty$ the groups of processors $\{1,2\}$ and $\{3\}$ of the model $X_{c}^{(3)}$ become asymptotically independent, so the processor 3 will move with the average speed $\lambda_{3}$.

Items 3 and 4 can be considered in a similar way. Note the item 3 consists of two subcases: $\lambda_{1}>\lambda_{3}>\lambda_{2}$ and $\lambda_{3}>\lambda_{1}>\lambda_{2}$. We omit details.

\section{Explicit construction of Lyapunov function}

In this section we prove the item 1 of Theorem 5 . Recall that our key assumption here is

$$
\lambda_{1}<\lambda_{2}, \quad \lambda_{1}<\lambda_{3}
$$

Main idea is to prove that the Markov chain $Y(n)$ is ergodic. To do this we apply the Foster-Lyapunov criterion (see Theorem 17 in Appendix). As in the case of Corollary 4 ergodicity of $Y(n)$ implies that $v_{j}^{*}=\lambda_{1}, j=1,2,3$.

\subsection{Transition probabilities}

Consider the embedded Markov chain $Y(n)$. A stochastic dynamics produced by this Markov chain consists of two components: transitions generated by the free dynamics and transitions generated by roll-backs. For each transition probability $p_{\alpha \beta}, \alpha \neq \beta$, we have the following representation:

$$
p_{\alpha \beta}=s_{\alpha \beta}+r_{\alpha \beta}
$$

where $s_{\alpha \beta} \geq 0$ corresponds to a transition $\alpha \rightarrow \beta$ which occurs due to the free dynamics and $r_{\alpha \beta}$ corresponds to a roll-back transition $\alpha \rightarrow \beta$.

Taking into account the remark at the end of Subsection 2.2, without loss of generality we assume that the time is rescaled in such way that $Z=1$. This slightly simplifies notation for transition probabilities. For example, free dynamics transitions (4)-(5) are equal to $\lambda_{2}, \lambda_{3}$ and $\lambda_{1}$ correspondingly. On the next figure we show all non-zero transitions $\alpha \rightarrow \beta,(\alpha \neq \beta)$. It is true, of course, that $p_{\alpha \alpha}=1-\sum_{\beta \neq \alpha} p_{\alpha \beta}$, but it is useless to put this 
information on the picture. Below we give explicit form of rollback transition probabilities:

$$
\begin{array}{rlrl}
1 & \rightarrow 2: & r_{y z} & =\beta_{12} \quad \text { for } 0<y_{2} \\
2 \rightarrow 3: & r_{y z} & =\beta_{23} \quad \text { for } y_{2}<y_{3} \\
1 \rightarrow 2 \rightarrow 3: & r_{y z}=\left\{\begin{aligned}
\beta_{12}\left(1-b_{2}\right)^{z_{3}} b_{2}, & z_{3}<y_{3} \\
\beta_{12}\left(1-b_{2}\right)^{y_{3}}, & z_{3}=y_{3}
\end{aligned} \text { for } 0<y_{3} \leq y_{2}\right. \\
1 \rightarrow 2 \rightarrow 3: & r_{y z}=\left\{\begin{array}{lll}
\beta_{12}\left(1-b_{2}\right)^{z_{3}} b_{2}, & z_{3} \leq y_{2} \\
\beta_{12}\left(1-b_{2}\right)^{y_{2}+1}, & z_{3}=y_{3}
\end{array} \text { for } 0<y_{2}<y_{3}\right.
\end{array}
$$

where $y=\left(y_{2}, y_{3}\right), z=\left(z_{2}, z_{3}\right)$.

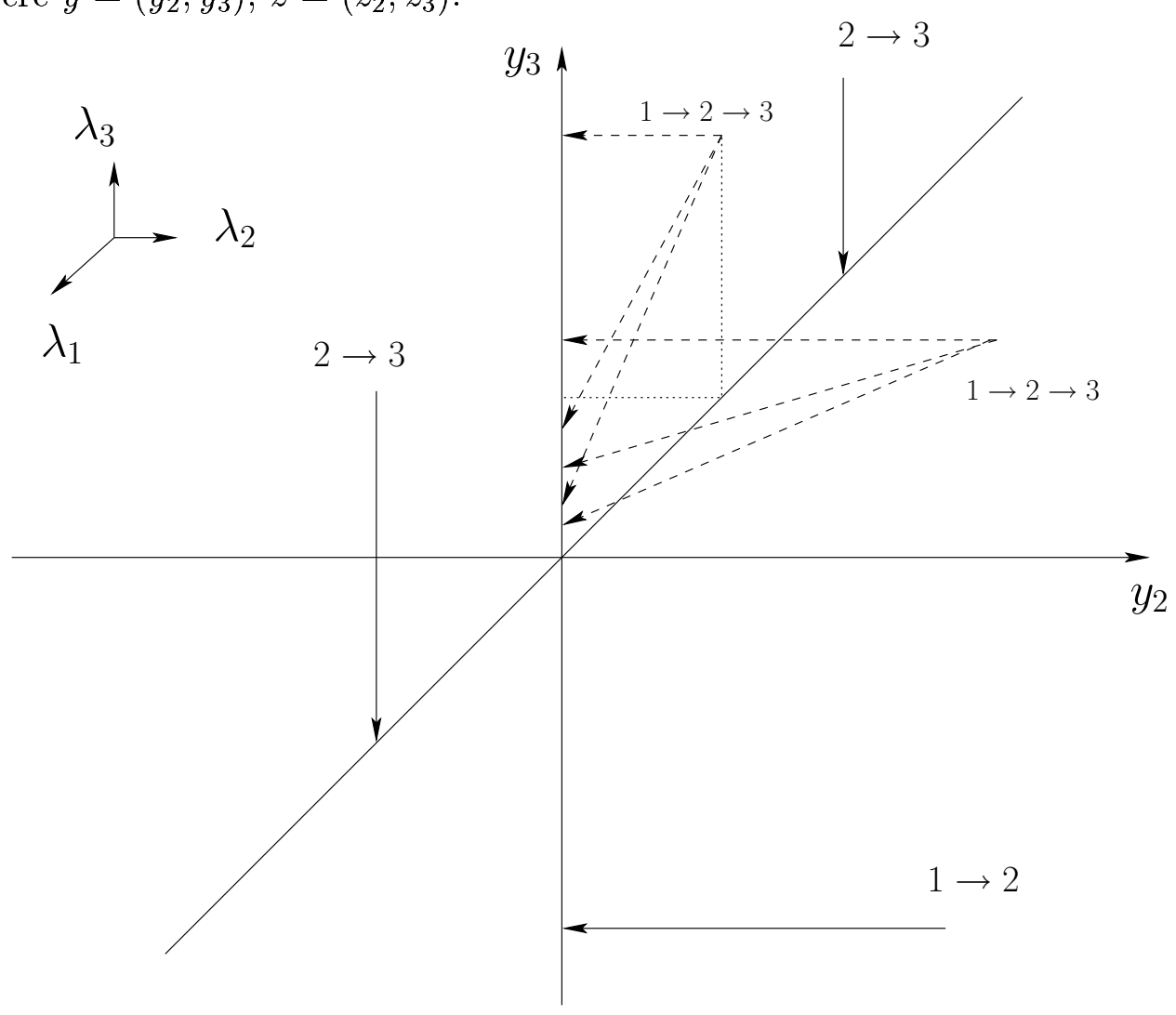

\subsection{Contour of level 1}

On the plain $\mathrm{Oy}_{2} y_{3}$ let us consider an ellipse

$$
e\left(y_{2}, y_{3}\right)=a y_{2}^{2}+b\left(y_{2}-y_{3}\right)^{2}=1, \quad a>0, b>0
$$


and draw a tangent line to it with normal vector $(-\Delta, 1)$. Evidently, there exist two tangent lines with the same normal vector $(-\Delta, 1)$. If $\Delta>0$ is sufficiently large then one of this tangent line touchs the ellipse at some point $T_{3}$ of the domain $y_{2}<0, y_{3}<0$. Take a segment on this line from the point $T_{3}$ to a point $K_{3}=\left(0, u_{3}\right)$ of intersection with coordinate axis $\mathrm{Oy}_{3}$. Now let us draw tangent lines to the ellipse corresponding to a normal vector $(1,-\Delta)$. If $\Delta>0$ is sufficiently large, then one of these lines touches the ellipse at some point $T_{2}$ of the domain $y_{3}<0$. Let us take this tangent line and fix a segment on it from the point $T_{2}$ to a point $K_{2}=\left(u_{2}, 0\right)$ of intersection with coordinate axis $O y_{2}$. It is evident that $\left[K_{2} K_{3}\right]=\mathbf{R}_{+}^{2} \cap\left\{\left(y_{2}, y_{3}\right): y_{2} / u_{2}+y_{3} / u_{3}=1\right\}$.

Let us consider now a closed contour $L$, consisting of subsequently joined segment $K_{3} K_{2}$, segment $K_{2} T_{2}$, arc $T_{2} T_{3}$ of the ellipse and segment $T_{3} K_{3}$. This countour has the following property: any ray of the form $\{c v, c>0\}$, where $v \in \mathbf{R}^{2}, v \neq 0$, has exactly one common point with the contour $L$.

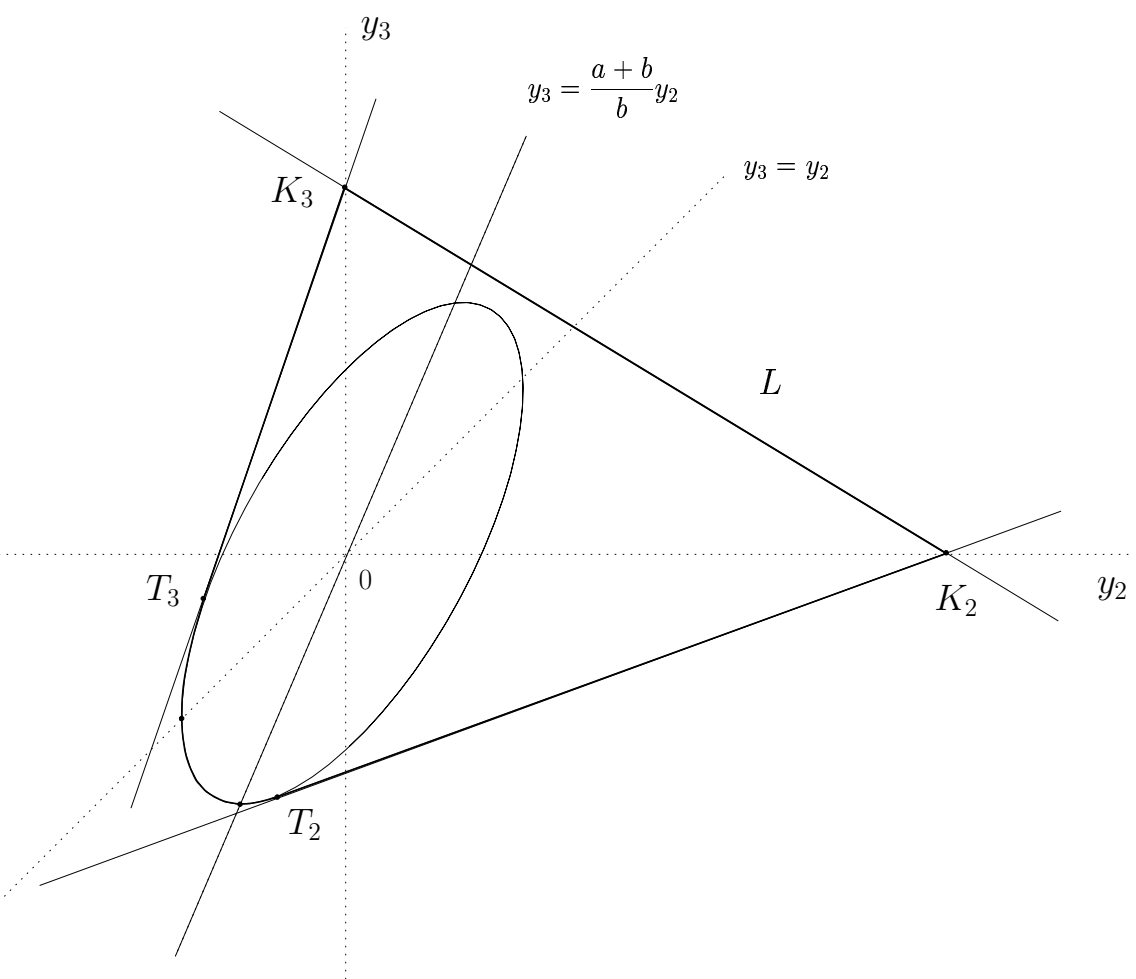

Denote by $n(y)$ a vector of unit length which is an outer normal vector to the contour $L$ at the point $y \in L$. The vector function $n(y)$ is well defined at all points of $L$ except $K_{2}$ and $K_{3}$ and, moreover, this function is continuous on $L$ except the points $K_{2}$ and $K_{3}$. A behaviour of the function $n(y)$ on the arc $T_{2} T_{3}$ is of main interest: 


$$
n(y)=\frac{\nabla e(y)}{\|\nabla e(y)\|}, \quad \nabla e(y)=2\left(a y_{2}+b\left(y_{2}-y_{3}\right),-b\left(y_{2}-y_{3}\right)\right), \quad y \in T_{2} T_{3} \subset L
$$

It is easy to see that $n(y)=n\left(T_{2}\right)$ for $y \in\left(K_{2} T_{2}\right], n(y)=n\left(T_{3}\right)$ for $y \in\left[T_{3} K_{3}\right)$ and

$$
n(y)=\left(u_{2}^{-1}, u_{3}^{-1}\right) \quad y \in\left(K_{3} K_{2}\right)
$$

For the sequel it is important to point out the following points of the $\operatorname{arc} T_{2} T_{3}: y^{(3)}=$ $\left(-a^{-1 / 2},-a^{-1 / 2}\right)$ and $y^{(2)},\left\{y^{(2)}\right\}=T_{2} T_{3} \cap\left\{y_{3}^{(2)}=\frac{a+b}{b} y_{2}^{(2)}\right\}$. It is easy to check that

$$
n\left(y^{(2)}\right)\left\|O y_{3}, \quad n\left(y^{(3)}\right)\right\| O y_{2}
$$

Evidently, the both points belong to the domain $\left\{y_{2}<0, y_{3}<0\right\}$.

\section{Lemma 6.}

The function $n(y)$ has the following properties:

- $\langle n(y), y\rangle \neq 0 \forall y \in L \backslash\left\{K_{2}, K_{3}\right\}$

- If $\Delta>0$ is sufficiently large then there exist continuous functions $c_{2}(y)$ and $c_{3}(y)$ such that

$$
\begin{gathered}
c_{2}\left(T_{2}\right)=c_{3}\left(T_{3}\right)=1, \quad c_{2}\left(T_{3}\right)=c_{3}\left(T_{2}\right)=0, \quad c_{2}(y)>0, c_{3}(y)>0 \quad y \in\left(T_{2}, T_{3}\right) \\
n(y)=c_{2}(y) n\left(T_{2}\right)+c_{3}(y) n\left(T_{3}\right), \quad y \in\left(T_{2}, T_{3}\right) .
\end{gathered}
$$

- $\langle n(y),(0,-1)\rangle<0$ if $y_{2}<0, y_{3}>y_{2}$, and $\langle n(y),(-1,0)\rangle<0$ if $y_{2}>0, y_{3}<0$.

\subsection{Definition of function $\varphi$}

For any point $\left(y_{2}, y_{3}\right) \in \mathbf{R}^{2} \backslash\{0\}$ define $\varphi\left(y_{2}, y_{3}\right)>0$ such that

$$
\frac{\left(y_{2}, y_{3}\right)}{\varphi\left(y_{2}, y_{3}\right)} \in L
$$

For $\left(y_{2}, y_{3}\right)=0$ we put $\varphi(0,0)=0$. The function $\varphi\left(y_{2}, y_{3}\right)$ is well-defined and has the following properties: 
- $\varphi: \mathbf{R}^{2} \rightarrow \mathbf{R}_{+}$(positivity)

- $\varphi\left(r y_{2}, r y_{3}\right)=r \varphi\left(y_{2}, y_{3}\right), r>0$, (homogeneity)

- $L=\{y: \varphi(y)=1\}$.

To any point $y=\left(y_{2}, y_{3}\right)$ we put in correspondance a point $y^{*}:=\frac{y}{\varphi(y)} \in L$. Therefore, $\varphi\left(y^{*}\right)=1$.

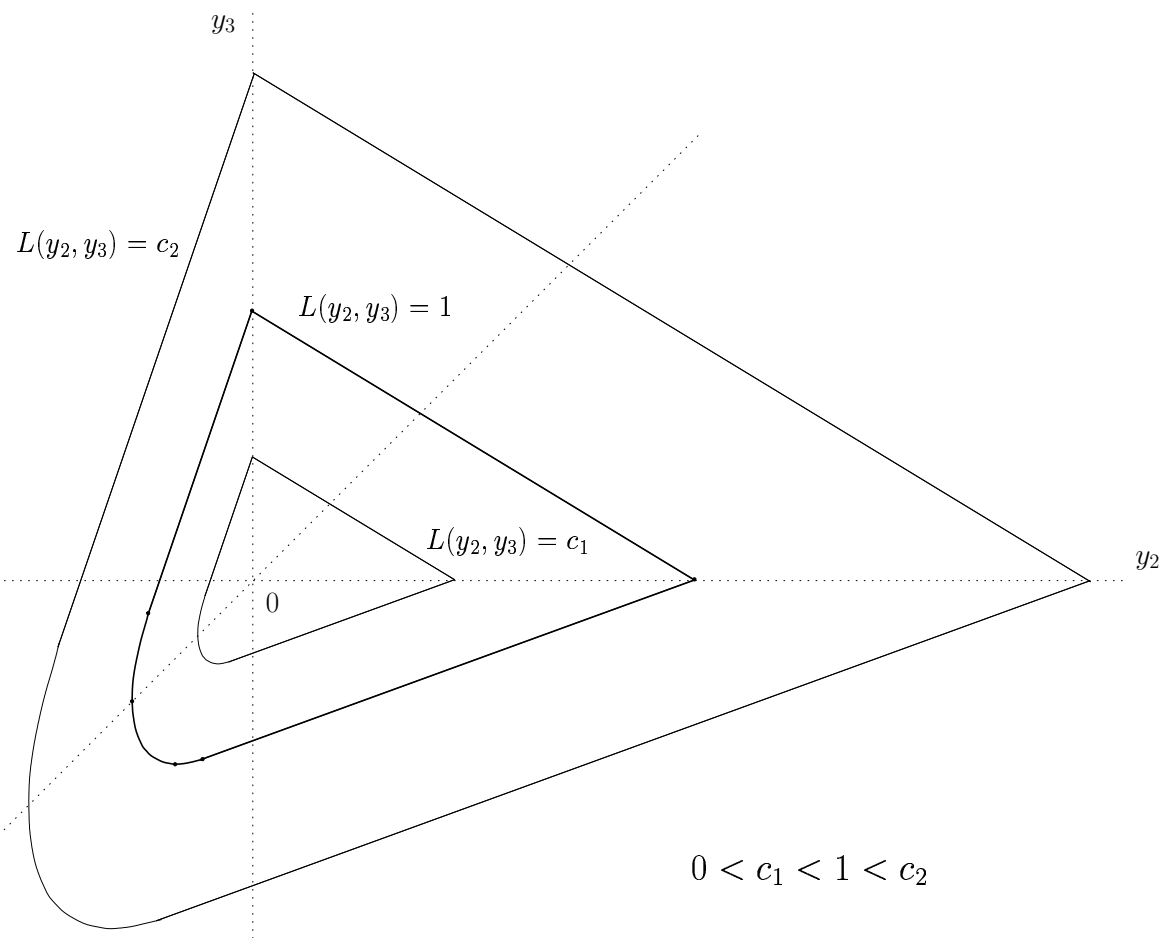

\section{Lemma 7.}

- A gradient $\nabla \varphi(y)$ exists at all points except that $y$ for which $y^{*}=K_{2}$ or $K_{3}$ and, moreover, the gradient is constant on rays of the form $\{c v, c>0\}, v \in \mathbf{R}^{2}$ :

$$
\nabla \varphi(y)=\frac{n\left(y^{*}\right)}{\left\langle y^{*}, n\left(y^{*}\right)\right\rangle}
$$

- Let $y=\left(y_{2}, y_{3}\right)$ be such that $y^{*} \in T_{2} T_{3}$. Then

$$
\left|\varphi(w)-\left\langle\nabla \varphi\left(y^{*}\right), w\right\rangle\right| \leq \frac{\text { const }}{\varphi(y)}\|w-y\|^{2}
$$

In other words, in a neighbourhood of the point $y$ the function $\varphi$ can be approximated by the linear function $\left\langle\nabla \varphi\left(y^{*}\right), \cdot\right\rangle$. 
In particular, $\varphi(y)=\left\langle\nabla \varphi\left(y^{*}\right), y\right\rangle$.

Proof of Lemma 7 is a straightforward computation.

\subsection{Modification of the principle of local linearity}

For any state $\alpha$ define a set $T_{\alpha}=\left\{\beta: p_{\alpha \beta}>0\right\}$. Recall decomposition (7) and define $F_{\alpha}=\left\{\beta: s_{\alpha \beta}>0\right\}$ and $R_{\alpha}=\left\{\beta: r_{\alpha \beta}>0\right\}$. It is evident that $T_{\alpha}=F_{\alpha} \cup R_{\alpha}$. The most simple case is $F_{\alpha} \cup R_{\alpha}=\varnothing$. The case $F_{\alpha} \cup R_{\alpha} \neq \varnothing$ can be reduced to the previous one by a dilatation of the state space. Thus we assume that $F_{\alpha} \cup R_{\alpha}=\varnothing$ and consider events $\left\{Y(n+1) \in F_{\alpha}\right\}$ and $\left\{Y(n+1) \in R_{\alpha}\right\}$. On the set $\{\omega \in \Omega: Y(n, \omega)=\alpha\}$ we have $I_{\left\{Y(n+1) \in F_{\alpha}\right\}}(\omega)+I_{\left\{Y(n+1) \in R_{\alpha}\right\}}(\omega) \equiv 1$. Hence,

$$
\begin{aligned}
\mathrm{E}(f(Y(n+1)) \mid Y(n)=\alpha)-f(\alpha)= & \mathrm{E}\left((f(Y(n+1))-f(y)) I_{\left\{Y(n+1) \in F_{\alpha}\right\}} \mid Y(n)=\alpha\right)+ \\
& +\mathrm{E}\left((f(Y(n+1))-f(y)) I_{\left\{Y(n+1) \in R_{\alpha}\right\}} \mid Y(n)=\alpha\right)
\end{aligned}
$$

It follows from definition of Markov chain $Y(n)$ (see Subsect. 7.1) that diameters $d_{\alpha}:=$ $\operatorname{diam} F_{\alpha}$ are uniformly bounded in $\alpha: d=\max _{\alpha} d_{\alpha}<+\infty$. Define a vector

$$
M_{F}(\alpha)=\mathrm{E}\left((Y(n+1)-\alpha) I_{\left\{Y(n+1) \in F_{\alpha}\right\}} \mid Y(n)=\alpha\right)=\sum_{\beta \in F_{\alpha}}(\beta-\alpha) p_{\alpha \beta} .
$$

This is an analogue of a notion of mean jump (see (12) in Appendix). In the next subsection we shall need the following modification of the principle of local linearity from [1] (see also Subsect. A.2 in Appendix).

Lemma 8. Assume that the following condition holds

$$
\inf _{l} \sup _{\tilde{\alpha} \in \mathbf{R}^{n},\|\tilde{\alpha}-\alpha\| \leq d_{\alpha}}|f(\tilde{\alpha})-l(\tilde{\alpha})|<\varepsilon,
$$

where inf is taken over all linear functions $l$. We state that from inequality

$$
f\left(\alpha+M_{F}(\alpha)\right)-f(\alpha)<-5 \varepsilon
$$

it follows that the following inequality

$$
\mathrm{E}\left((f(Y(n+1))-f(\alpha)) I_{\left\{Y(n+1) \in F_{\alpha}\right\}} \mid Y(n)=\alpha\right)<-\varepsilon
$$


holds.

Proof of this statement repeats the proof of principle of local linearity presented in [1] and, thus, is omitted.

\subsection{Proof of Foster condition}

The validity of the Foster condition will follow from several auxilary lemmas dealing with the following different domains of the state space:

$$
\begin{aligned}
E_{-} & :=\left\{y=\left(y_{2}, y_{3}\right): \min \left(y_{2}, y_{3}\right)<0\right\} \\
E_{1} & :=\left\{y=\left(y_{2}, y_{3}\right): y_{2}>0, y_{3}>0\right\} \\
E_{1,2} & :=\left\{y_{2}>0, y_{3}=0\right\} \\
E_{1,3} & :=\left\{y_{2}=0, y_{3}>0\right\} .
\end{aligned}
$$

Lemma 9. Consider domain $E_{-}=\left\{y=\left(y_{2}, y_{3}\right): \min \left(y_{2}, y_{3}\right)<0\right\}$. There exists $C_{9}>0$, such that if $\varphi(y)>C_{9}$, then

a)

$$
\mathrm{E}\left((\varphi(Y(n+1))-\varphi(y)) I_{\left\{Y(n+1) \in R_{y}\right\}} \mid Y(n)=y\right) \leq 0,
$$

b) there exists $\varepsilon>0$ such that

$$
\varphi\left(y+M_{F}(y)\right)-\varphi(y)<-5 \varepsilon
$$

Proof. It is evident that the vector

$$
M_{F}(y)=\left(\lambda_{2}-\lambda_{1}, \lambda_{3}-\lambda_{1}\right)
$$

is constant (does not depend on $y$ ). Since the vector $n\left(T_{2}\right)$ is co-directed with the vector $(1,-\Delta)$ and the vector $n\left(T_{3}\right)$ is co-directed with the vector $(-\Delta, 1)$ and the conditions $\lambda_{2}>\lambda_{1}, \lambda_{3}>\lambda_{1}$ hold, we can find a large $\Delta_{1}>0$ such that

$$
\left\langle M_{F}(y), n\left(T_{2}\right)\right\rangle<0, \quad\left\langle M_{F}(y), n\left(T_{3}\right)\right\rangle<0, \quad \forall \Delta>\Delta_{1} .
$$

Fix this $\Delta_{1}$. Hence, by Lemma 6 there exists $\varepsilon>0$ such that

$$
\left\langle M_{F}(y), n(y)\right\rangle<-6 \varepsilon \quad \text { if } \min \left(y_{2}, y_{3}\right)<0 \text {. }
$$


Put $w=y+M_{F}(y)$ and consider

$$
\begin{aligned}
\varphi(w)-\varphi(y) & =\varphi(w)-\left\langle\nabla \varphi\left(y^{*}\right), y\right\rangle \\
& =\varphi(w)-\left\langle\nabla \varphi\left(y^{*}\right), w\right\rangle+\left\langle\nabla \varphi\left(y^{*}\right), w-y\right\rangle \\
& =\varphi(w)-\left\langle\nabla \varphi\left(y^{*}\right), w\right\rangle+\left\langle\nabla \varphi\left(y^{*}\right), M_{F}(y)\right\rangle
\end{aligned}
$$

By (8) for any given $\varepsilon>0$ we can choose $C_{0}>0$ such that

$$
\left|\varphi\left(y+M_{F}(y)\right)-\left\langle\nabla \varphi\left(y^{*}\right), M_{F}(y)\right\rangle\right| \leq \frac{\text { const }}{\varphi(y)} d^{2} \leq \varepsilon \quad \text { if } \varphi(y) \geq C_{0}
$$

Now the item b) of the lemma easily follows.

Let us prove the item a) of the lemma. Note that in the domain $y_{3}>y_{2}, y_{2}<0$ a rollback decreases coordinate $y_{3}:\left(y_{2}, y_{3}\right) \rightarrow\left(y_{2}^{\prime}, y_{3}^{\prime}\right)=\left(y_{2}, y_{2}\right)$. From geometrical properties of level sets of function $\varphi$ and item 3 of Lemma 6 it follows that any transition generated by a rollback decreases a value of the function $\varphi: \varphi\left(\left(y_{2}, y_{3}\right)\right)<\varphi\left(\left(y_{2}^{\prime}, y_{3}^{\prime}\right)\right)$. In the domain $y_{3}<0, y_{2}>0$ a rollback has the following form: $\left(y_{2}, y_{3}\right) \rightarrow\left(y_{2}^{\prime}, y_{3}^{\prime}\right)=\left(0, y_{3}\right)$. For similar reasons we again have $\varphi\left(\left(y_{2}, y_{3}\right)\right)<\varphi\left(\left(y_{2}^{\prime}, y_{3}^{\prime}\right)\right)$. In the domain $y_{3} \leq y_{2}<0$ there is no rollback. Now the item a) easily follows.

Lemma 10. Consider domain: $E_{1}=\left\{y=\left(y_{2}, y_{3}\right): y_{2}>0, y_{3}>0\right\}$.

1. The conditional expectation

$$
\mathrm{E}\left((\varphi(Y(n+1))-\varphi(y)) I_{\left\{Y(n+1) \in F_{y}\right\}} \mid Y(n)=y\right)=\left\langle\left(u_{2}^{-1}, u_{3}^{-1}\right), M_{F}(y)\right\rangle
$$

does not depend on $y$.

2. There exist constants $C_{10}, \gamma_{10}>0$ such that

$$
\mathrm{E}\left((\varphi(Y(n+1))-\varphi(y)) I_{\left\{Y(n+1) \in R_{y}\right\}} \mid Y(n)=y\right) \leq-\gamma_{10} \varphi(y) \quad \text { if } \varphi(y)>C_{10}
$$

Proof. The second statement follows from the fact that in this domain $\varphi(y)=\left\langle\left(u_{2}^{-1}, u_{3}^{-1}\right), y\right\rangle$, and the vector $M_{F}(y)$ does not depend on $y$.

Let us prove (9). Fix some level set $\left\{y_{2} / u_{2}+y_{3} / u_{3}=C\right\} \cap\left\{y_{2}>0, y_{3}>0\right\}$ and consider an action of rollbacks. We have three different situations.

a) Let $y$ be such that $y_{2} \geq y_{3}>0$. It follows that $y_{2} \geq \frac{C}{\frac{1}{u_{2}}+\frac{1}{u_{3}}}$. With probability $\beta_{12}$ we have a rollback of the following form $\left(y_{2}, y_{3}\right) \rightarrow\left(0, y_{3}^{\prime}\right)$ where $0 \leq y_{3}^{\prime} \leq y_{3}$. Hence the 
function $\varphi(y)$ will decrease at least on the value

$$
\frac{C}{\frac{1}{u_{2}}+\frac{1}{u_{3}}}: u_{2}=\frac{C}{1+u_{2} / u_{3}}
$$

and the conditional mean $\mathrm{E}\left((\varphi(Y(n+1))-\varphi(y)) I_{\left\{Y(n+1) \in R_{y}\right\}} \mid Y(n)=y\right)$ will not exceed the value $-\beta_{12}\left(1+u_{2} / u_{3}\right)^{-1} C$.

b) Let $y$ be such that $0<\frac{1}{2} y_{3} \leq y_{2}<y_{3}$. It follows that $y_{2} \geq \frac{C}{2\left(\frac{1}{u_{2}}+\frac{1}{u_{3}}\right)}$. With probability $\beta_{12}$ we have a rollback $\left(y_{2}, y_{3}\right) \rightarrow\left(0, y_{3}^{\prime}\right)$ where $0 \leq y_{3}^{\prime} \leq y_{3}$ and with probability $\beta_{23}$ we have a rollback $\left(y_{2}, y_{3}\right) \rightarrow\left(y_{2}, y_{2}\right)$. The both of them will decrease the function $\varphi$. But the first rollback will decrease it at least on the value $\frac{C}{2\left(1+u_{2} / u_{3}\right)}$. So we conclude that the above conditional mean will not exceed the value $-\frac{1}{2} \beta_{12}\left(1+u_{2} / u_{3}\right)^{-1} C$.

c) Now let $y$ be such that $0<y_{2} \leq \frac{1}{2} y_{3}$. It follows that $y_{3}-y_{2} \geq K(C)$ where

$$
K(C):=\frac{1}{2} \cdot \frac{C}{\left(\frac{1}{2 u_{2}}+\frac{1}{u_{3}}\right)}=\frac{C u_{3}}{u_{3} / u_{2}+2} .
$$

With probability $\beta_{12}$ we have a rollback $\left(y_{2}, y_{3}\right) \rightarrow\left(0, y_{3}^{\prime}\right), 0 \leq y_{3}^{\prime} \leq y_{3}$, which decreases the function $\varphi$, and with probability $\beta_{23}$ we have a rollback $\left(y_{2}, y_{3}\right) \rightarrow\left(y_{2}, y_{2}\right)$, which decreases the value of the function $\varphi$ at least on $K(C) / u_{3}$. Hence the conditional expectation will decrease at least on the value $-\beta_{23} K(C) / u_{3}=-\beta_{23}\left(u_{3} / u_{2}+2\right)^{-1} C$.

Lemma 11. Consider the cases when $y$ belongs to the axes: $y \in E_{1,3}, y \in E_{2,3}$. Here

$$
\mathrm{E}\left((\varphi(Y(n+1))-\varphi(y)) I_{\left\{Y(n+1) \in R_{y}\right\}} \mid Y(n)=y\right) \leq-\gamma_{11} \varphi(y)
$$

and

$$
\mathrm{E}\left((\varphi(Y(n+1))-\varphi(y)) I_{\left\{Y(n+1) \in F_{y}\right\}} \mid Y(n)=y\right)
$$

does not depend on $y$, where $y \in\left\{y: \varphi(y)>C_{11}\right\}$.

Proof. We consider in details the case $E_{1,3}=\left\{y_{2}=0, y_{3}>0\right\}$. We start with the free dynamics. The following transition

$$
\left(0, y_{3}\right) \rightarrow\left(y_{2}^{\prime}, y_{3}^{\prime}\right)=\left(-1, y_{3}-1\right) \in E_{3}=\left\{y_{2}<0, y_{3}>y_{2}\right\}
$$

occurs with probability $\lambda_{1}$. It is easy to see that for $y_{3}>C_{11}$ values of the function $\varphi(\cdot)$ 
in the both points $\left(0, y_{3}\right)$ and $\left(-1, y_{3}-1\right)$ concide with values of linear function $\left\langle n\left(T_{3}\right), \cdot\right\rangle$.

With probability $\lambda_{2}$ we have a transition

$$
\left(0, y_{3}\right) \rightarrow\left(y_{2}^{\prime}, y_{3}^{\prime}\right)=\left(1, y_{3}\right) \in E_{1}=\left\{y_{2}>0, y_{3}>0\right\}
$$

and with probability $\lambda_{3}$ we have a transition

$$
\left(0, y_{3}\right) \rightarrow\left(y_{2}^{\prime}, y_{3}^{\prime}\right)=\left(0, y_{3}+1\right) \in E_{3}
$$

Evidently, that in all these points $\left(0, y_{3}\right),\left(1, y_{3}\right)$ and $\left(0, y_{3}+1\right)$ values of $\varphi(\cdot)$ coincide with values of a linear function $\left\langle n_{1}, \cdot\right\rangle$. Hence,

$$
\begin{aligned}
\mathrm{E}\left((\varphi(Y(n+1))-\varphi(y)) I_{\left\{Y(n+1) \in F_{y}\right\}} \mid Y(n)=y\right)= & \lambda_{1}\left\langle n\left(T_{3}\right),(-1,-1)\right\rangle \\
& +\lambda_{2}\left\langle n_{1},(1,0)\right\rangle+\lambda_{3}\left\langle n_{1},(0,1)\right\rangle .
\end{aligned}
$$

Since the r.h.s. does not depend on $y$ we get the second statement of the lemma.

Due to a rollback the Markov chain $Y(n)$ goes from the state $\left(0, y_{3}\right) \in E_{1,3}$ to a state $(0,0)$ with probability $\beta_{23}$. Since values of $\varphi(\cdot)$ at these two points can be calculated by using the linear function $\left\langle n_{1}, \cdot\right\rangle$, the rollback decreases the value of $\varphi$ on $C$, where $C=\varphi\left(\left(0, y_{3}\right)\right)$. The first statement of the lemma is proved.

The case of the domain $E_{1,3}=\left\{y_{2}>0, y_{3}=0\right\}$ is similar.

Lemma 12. For any $C_{0}>0$

$$
\sup _{y: \varphi(y) \leq C_{0}} \mathrm{E}\left(\varphi\left(Y_{t+1}\right) \mid Y_{t}=y\right)<+\infty
$$

Proof. This statement follows from the fact that jumps from any fixed neighbourhood of $(0,0)$ are bounded and the fact that the function $\varphi$ is continuous. 


\section{Conclusions, conjectures and perspectives}

\subsection{Decomposition into groups}

We shall always assume that all $\lambda_{1}, \ldots, \lambda_{N}$ are different. Define a function

$$
\ell(m):=\min _{i \leq m} \lambda_{i}
$$

Evidently, this function has the following property:

$$
\ell(1)=\lambda_{1} \geq \cdots \geq \ell(m) \geq \ell(m+1) \geq \cdots \geq \min \left(\lambda_{1}, \ldots, \lambda_{N}\right)=\ell(N) .
$$

Level sets of function $\ell$ genetate a partition of the set $\{1, \ldots, N\}$. Namely, there exists a sequence $j_{1}=1<j_{2}<\cdots<j_{K}<j_{K+1}=N+1$ such that the set of all processors can be divided into several nonintersecting groups

$$
\begin{gathered}
\{1, \ldots, N\}=\bigcup_{k=1, K} G_{k}, \\
G_{k}:=\left\{j_{k}, j_{k}+1, \ldots, j_{k+1}-1\right\}, \quad \ell\left(j_{k}-1\right)>\ell\left(j_{k}\right)=\cdots=\ell\left(j_{k+1}-1\right)>\lambda_{j_{k+1}} .
\end{gathered}
$$

Remark 13. An equivalent description of the group is possible. We say, for example, that $1,2, \ldots, k$ is a group if

$$
\lambda_{1} \leq \min \left(\lambda_{2}, \ldots, \lambda_{k}\right), \quad \lambda_{1}>\lambda_{k+1}
$$

\subsection{Long-time behaviour of the groups}

Taking into account Theorems 3 and 5 and the above notion of groups of processors we put forward the following Conjecture.

Conjecture 14. Assume that all $\lambda_{1}, \ldots, \lambda_{N}$ are different. For any $j$ the following limit $v_{j}^{*}=\lim _{t \rightarrow+\infty} \frac{x_{j}(t)}{t}$ exists and $v_{j}^{*}=\ell(j)$.

So by this conjecture $v_{j}^{*}=\ell\left(j_{k}\right)$ for $j \in G_{k}$. If for some $k$ the group $G_{k}$ consists of more than one processor we may say that the processors of the group $G_{k}$ are synchronized.

Remark 15 (On monotone cases). If $\lambda_{1}<\cdots<\lambda_{N}$ then $v_{j}^{*}=\lambda_{1}$ for any $j$. If $\lambda_{1}>\cdots>\lambda_{N}$ then for all $j$ we have $v_{j}^{*}=\lambda_{j}$. 
Let us discuss briefly perspectives of rigorous proof of the above Conjecture for large values of $N$. In fact, we have already prove this conjectures for wide class of cascade models.

Theorem 16. Assume that all $\lambda_{1}, \ldots, \lambda_{N}$ are different and a partition (10) of the set of processors $\{1, \ldots, N\}$ is such that $\left|G_{k}\right| \leq 3$ for all $k$. Then the limits $v_{j}^{*}=\lim _{t \rightarrow+\infty} \frac{x_{j}(t)}{t}$ exist and $v_{j}^{*}=\ell(j)$.

Proof of this statement is just a combination of the result of Theorem 5 (item 1) and arguments of the proof of items 2-4 of Theorem 5. We will not go into details.

So the key to the proof of Conjecture consists in generalization of item 1 of Theorem 5 . As we see from Section 7 one of possible way of such generalization is an explicit construction of Foster-Lyapunov function in high dimensions. This seems to be a dificult technical problem which is out of the purposes of this paper.

\section{A Appendix}

Let $\left(\xi_{n}, n=0,1, \ldots\right)$ be a countable irreducible aperiodic Markov chain with the state space $\mathcal{A}$.

\section{A.1 Criteria}

We use the following Foster criterion.

Theorem 17 ([1]). The Markov chain $\xi_{n}$ is ergodic if and only if there exists a positive function $f(\alpha), \alpha \in \mathcal{A}$, a number $\varepsilon>0$ and a finite set $A \in \mathcal{A}$ such that 1)

$$
\mathrm{E}\left(f\left(\xi_{n+1}\right) \mid \xi_{n}=y\right)-f(y)<-\varepsilon
$$

for all $y \notin A$,

2) $\mathrm{E}\left(f\left(\xi_{n+1}\right) \mid \xi_{n}=y\right)<+\infty$ for all $y \in A$.

The following theorem give a criterion of transiency.

Theorem 18 ([1]). The Markov chain is transient, if and only if there exists a positive function $f(\alpha)$ and a set $A$ such that the following inequalities are fulfilled

$$
\mathrm{E}\left(f\left(\xi_{m+1}\right) \mid \xi_{m}=\alpha_{i}\right)-f\left(\alpha_{i}\right) \leq 0, \quad \forall \alpha_{i} \notin A
$$




$$
f\left(\alpha_{k}\right)<\inf _{\alpha_{j} \in A} f\left(\alpha_{j}\right), \quad \text { for at least one } \alpha_{k} \notin A \text {. }
$$

\section{A.2 Principle of local linearity}

From now we asume that the state space $\mathcal{A}$ is a subset of some $\mathbf{R}^{k}$. Define a vector of mean jump from the point $\alpha$

$$
M(\alpha)=\mathrm{E}\left(\left(\xi_{n+1}-\alpha\right) \mid \xi_{n}=\alpha\right)=\sum_{\beta}(\beta-\alpha) p_{\alpha \beta}
$$

Assume that $d_{\alpha}:=\max _{\beta}\left\{|\beta-\alpha|: p_{\alpha \beta}>0\right\}<\infty$ for all $\alpha$.

The following principle of local linearity was proved in [1].

Lemma 19. Assume that at some point $\alpha$ the following condition

$$
\inf _{l} \sup _{\tilde{\alpha} \in \mathbf{R}^{n},\|\tilde{\alpha}-\alpha\| \leq d_{\alpha}}|f(\tilde{\alpha})-l(\tilde{\alpha})|<\varepsilon
$$

holds, where inf is taken over all linear functions $l$. Then

$$
f(\alpha+M(\alpha))-f(\alpha)<-5 \varepsilon \quad \Longrightarrow \quad \mathrm{E}\left(f\left(\xi_{n+1}\right)-f(\alpha) \mid \xi_{n}=\alpha\right)<-\varepsilon .
$$

\section{References}

[1] Fayolle G., Malyshev V., Menshikov M., Topics on constructive countable Markov chains. Cambridge University Press, 1995.

[2] D.P. Bertsekas, J.N. Tsitsiklis, Parallel and Distributed Computation: Numerical Methods. Athena Scientific, Belmont, Mass. 1997.

[3] D. Mitra, I. Mitrani, Analysis and Optimum performance of two message-passing parallel processors synchronizhed by rollback. Performance Evaluation 7 (1987), 111-124

[4] V.K. Madisetti, J.C. Walrand and D.G. Messerschmitt, Asynchronous Algorithms for the ParaSimulation of Event-Driven Dynamical Systems, ACM Transactions on Modelling and Computer Simulation, Vol. 1, No 3, July 1991, Pages 244-274

[5] S.Yu. Popov, A.G. Greenberg, V.A. Malyshev, Stochastic models of massively parallel computation. Markov Processes and Related Fields, V.1, N4 (1995), 473-490. 
[6] A.G. Greenberg, S. Shenker, A.L. Stolyar, Asynchronous Updates in Large Parallel Systems. SIGMETRICS 96 5/96 PA, USA

[7] A. Gupta, I.F. Akyildiz, Fujimoto, Performance Analysis of Time Warp With Multiple Homogeneous Processors. IEEE Transactions On Software Engineering, Vol. 17, No. 10, October 1991, 1013.

[8] M. Gupta, A. Kumar, R. Shorey, Queueing Models and Stability of Message Flows in Distributed Simulators of Open Queueing Networks. Preprint.

[9] M. Gupta and A. Kumar, A Nonblocking Algorithm for the Distributed Simulation of FCFS Queueing Networks with Irreducible Markovian Routing. Preprint.

[10] A. Kumar and R. Shorey, Stability of Event Synchronisation in Distributed Discrete Event Simulation. Preprint.

[11] R. Shorey, A. Kumar, and K.M. Rege, Instability and Performance Limits of Distributed Simulators of Feedforward Queueing Networks. ACM Transactions on Modeling and Computer Simulation, Vol. 7, No. 2, April 1997, Pages 210-238.

[12] T.V. Voznesenskaya, Analysis of algorithms of time synchronisation for distributed simulation. Artificial intelligence (Donetsk), N2, 24-30 (2000) (in Russian).

[13] T.V. Voznesenskaya, Mathematical model of algorithms of synchronization of time for the distributed simulation,in L.N. Korolev (Eds.), "Program systems and tools": the Thematic collection of faculty VMiK of the Moscow State University N1: MAX Press, 56-66 (2000).

[14] D. Jefferson, A. Witkowski, An Approach to Performance Analysis of Time stamp-driven Synchronization Mechanisms. 1984 ACM0-89791-143-1 84, 008/0243

[15] I.F. Akyildiz, L. Chen, S.R. Dast, R.M. Fujimoto, R.F. Serfozo, Performance Analysis of Time Warp with Limited Memory. Performance Evaluation Review, Vol. 20, No. 1, June 1992

[16] Malyshev V., Manita A. Time synchronization problem. Rapport de recherche INRIA, N. 5204, 2004.

[17] A. Manita, V. Shcherbakov. Asymptotic analysis of particle system with mean-field interaction, arXiv:math.PR/0408372 (http://arxiv.org).

[18] Malyshev V.A., Manita A.D. Phase transitions in the time synchronization model. Probability Theory and Applications, Vol. 50, 2005 